\title{
Large-scale statistical study of Scanning Multichannel Microwave Radiometer (SMMR) data over Antarctica
}

\author{
Michel Fily and Jean-Pierre Benoist \\ Laboratoire de Glaciologie et de Géophysique de l'Environnement, 38402 Saint-Martin-d'Hères Cedex, France
}

\begin{abstract}
SMMR data over Antarctica have been statistically analysed for four different periods of 1 year (1981) and compared to geophysical data such as surface temperature, snow-accumulation rate and topography. The spatial variations of the microwave signature are stable with time. Although the ten channels are highly correlated, principal-component analysis reveals the importance of polarization and frequency. The difference between brightness temperatures at the two polarizations is found to be dependent on the atmospheric water-vapour fluxes over the ice sheet, which modify the temperature-accumulation ratio and therefore the snow stratification. The brightness-temperature gradient with frequency is related to the topography of the central plateau area. A more important subsidence over diverging areas could explain the different structure of the accumulated snow.
\end{abstract}

\section{INTRODUCTION}

Ground data are sparse in Antarctica because of the harsh environment and the small number of permanent scientific research stations on the central plateau. Therefore, remote sensing is a promising tool in the quest to improve our knowledge of surface characteristics, such as surface temperature or snow-accumulation rate, which are crucial for ice-sheet modelling.

Among satellite-borne sensors, particular attention has been paid to microwave radiometers because of the relative independence from sunlight and atmospheric conditions. Moreover, the penetration of microwaves in the dry snow provides information on its characteristics through some depth. The poor spatial resolution of the microwave data $(30-100 \mathrm{~km})$ does not hamper large-scale studies.

Past studies have revealed unpredicted low emissivity on the Greenland and Antarctic ice sheets (Gloersen and others, 1974). The cause has been attributed to scattering by snow grains (Chang and others, 1976). As the grain-size varies with age (Gow, 1969), the emissivity has been related to snow-accumulation rate (Zwally, 1977). Until now, studies have been mainly based on data from the Nimbus-5 Electrically Scanning Microwave Radiometer (ESMR) (Chang and others, 1980) and the Nimbus-6 Scanning Microwave Spectrometer (SCAMS) (Rotman and others, 1981, 1982). Data from the Nimbus-7 Scanning Multichannel Microwave Radiometer (SMMR) have been extensively used on sea ice (Gloersen and Campbell, 1988) and on snow-covered terrains (Kunzi and others, 1982), but little work has been done on the ice sheets (Rott, 1989; Zhang and others, 1989). The applications of microwave remote sensing of snow have been recently reviewed by Mätzler (1987).

The objectives of the present paper are to look at the statistical behavior of the Nimbus-7 SMMR brightness temperatures over the Antarctic ice sheet and then to relate them to geophysical data in order to obtain a better understanding of their spatial distribution.

The SMMR and geophysical data are described in section 2. Section 3 is devoted to the statistical study which is mainly based on a principal-component analysis. The aim is to determine how many parameters we can hope to obtain using the ten channels and which combinations are the most useful. Such studies have been done over the Arctic sea ice by Rothrock and others (1988). In section 4, we compare SMMR data to geophysical data (surface temperature, snow-accumulation rate, topography) by means of correlation and regression analysis. We then discuss some possible causes of the spatial pattern of microwave signatures over the ice sheet.

\section{DATA SETS}

\section{SMMR data}

The Nimbus-7 satellite (Madrid, 1978) was launched in October 1978 and operated successfully for almost 9 years, providing valuable long-term data sets for climaterelated studies. The SMMR instrument measures microwave radiation from the Earth's surface at five frequencies in both horizontal and vertical polarizations. Channel characteristics are given in Table 1 with their approximate ground resolution. The SMMR operates on 
Table 1. Characteristics of ten SMMR channels. $H$ and $V$ refer to horizontal and vertical polarization

\begin{tabular}{|c|c|c|c|c|c|c|c|c|}
\hline Number & 1 & 2 & 3 & 4 & 5 & 7 & 8 & 9 \\
\hline Polarization & $\mathrm{H}$ & V & $\mathrm{H}$ & V & $\mathrm{H}$ & $\mathrm{H}$ & V & $\mathrm{H}$ \\
\hline Frequency $(\mathrm{GHz})$ & 6.6 & & 10.7 & & 18.0 & 21.0 & & 37.0 \\
\hline Wavelength $(\mathrm{cm})$ & 4.5 & & 2.8 & & 1.7 & 1.4 & & 0.8 \\
\hline Resolution (km) & 150 & & 90 & & 60 & 50 & & 30 \\
\hline
\end{tabular}

alternate days, providing a global coverage of the Earth every $6 \mathrm{~d}$. At present, due to its high latitude, Antarctica is covered in $3 \mathrm{~d}$ except for the region poleward of $84^{\circ} \mathrm{S}$ which is not covered because of the orbital and instrumental geometry.

All channels have been operating successfully except for the $21 \mathrm{GHz}$ channel which has drifted a lot more than the other channels since the beginning of the operation. Data have been corrected for this channel following $\mathrm{Fu}$ and others (1988). Nevertheless, we neglected this channel for some applications. We used the Cell-All Tape data which are available from NASA's National Space Data Center, Central Data Services Facility, Greenbelt, Maryland (Fu and others, 1988). On these tapes, the ten-channel brightness temperatures have been binned and averaged in $150 \mathrm{~km} \times 150 \mathrm{~km}$ square cells.

Four different periods in 1981 were chosen for analysis: 22-27 January (day 22), 5-9 April (day 95), 10-14 July (day 191), 1-5 November (day 305). Geographical selection of data over the ice sheet was done on the basis of the Cell-All Tapes specifications. About 3000 observations (grid cells) are available per period.

The data were used as provided by NASA for the principal-component analysis. For correlation with geophysical data and contour plots, we interpolated these data on a grid similar to the one used for the geophysical data. The latter grid is centered on the South Pole and has a $100 \mathrm{~km} \times 100 \mathrm{~km}$ mesh size. Only the cells containing at least one SMMR observation were used.

Because of the low atmospheric water-vapour content over the Antarctic ice sheet (Zwally and Gloersen, 1977), the atmospheric effects were ignored.

\section{Geophysical data}

Elevation, mean surface temperature and snow-accumulation rate (Fig. 1) were provided by the Meteorology Department, University of Melbourne, in a digital format. Surface elevations were digitized from Drewry's (1983) map. For accumulation rate and temperature, preliminary maps from the Scott Polar Research Institute, Cambridge, were used together with many other sources (Budd and Smith, 1982; Jenssen and others, 1985; Radok and others, 1986). The greatest uncertainty lies in the snow-accumulaton rates and some differences appear with other compilations (Giovinetto and Bull, 1987).

Although all these data are available for a $20 \mathrm{~km}$ and a
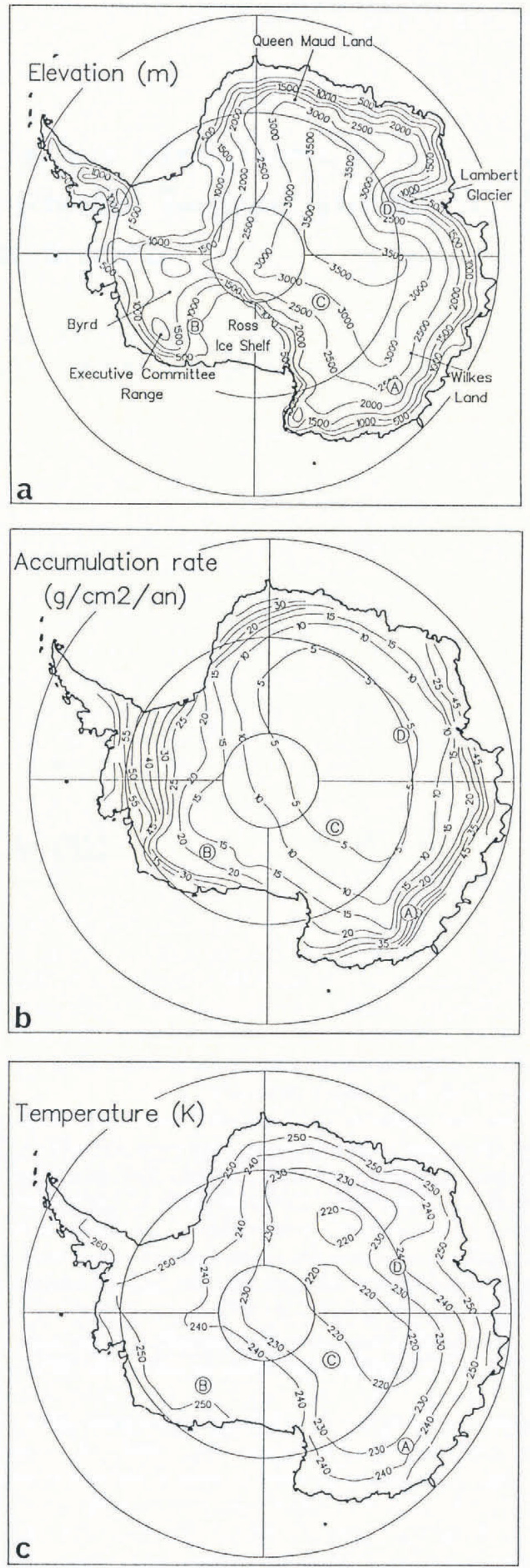

Fig. 1. (a) Elevation (m) map of the Antarctic ice sheet; (b) Snow-accumulation rate $\left(\mathrm{g} \mathrm{cm}^{-2}\right.$ year $\left.^{-1}\right)$; (c) Mean annual surface temperature $(K)$. Some selected areas which we refer to in the text are labelled with letters. 


\begin{tabular}{|c|c|c|c|c|c|c|c|c|c|c|}
\hline 1 & 2 & 3 & 4 & 5 & 6 & 7 & 8 & 9 & 10 & Channel \\
\hline 1.0 & $\begin{array}{l}0.85 \\
1.0\end{array}$ & $\begin{array}{l}0.96 \\
0.85 \\
1.0\end{array}$ & $\begin{array}{l}0.85 \\
0.97 \\
0.91 \\
1.0\end{array}$ & $\begin{array}{l}0.90 \\
0.84 \\
0.98 \\
0.92 \\
1.0\end{array}$ & $\begin{array}{l}0.85 \\
0.93 \\
0.92 \\
0.99 \\
0.95 \\
1.0\end{array}$ & $\begin{array}{l}0.90 \\
0.84 \\
0.97 \\
0.92 \\
0.99 \\
0.95 \\
1.0\end{array}$ & $\begin{array}{l}0.82 \\
0.91 \\
0.88 \\
0.95 \\
0.90 \\
0.96 \\
0.90 \\
1.0\end{array}$ & $\begin{array}{l}0.90 \\
0.90 \\
0.95 \\
0.94 \\
0.96 \\
0.95 \\
0.97 \\
0.92 \\
1.0\end{array}$ & $\begin{array}{l}0.87 \\
0.96 \\
0.90 \\
0.97 \\
0.91 \\
0.97 \\
0.91 \\
0.94 \\
0.97 \\
1.0\end{array}$ & $\begin{array}{r}1 \\
2 \\
3 \\
4 \\
5 \\
6 \\
7 \\
8 \\
9 \\
10\end{array}$ \\
\hline
\end{tabular}

$100 \mathrm{~km}$ mesh size, we preferred the $100 \mathrm{~km} \times 100 \mathrm{~km}$ grid because it was precise enough for our large-scale study and closer to the SMMR cell size.

\section{STATISTICAL STUDY}

\section{Correlation and multiple-regression analysis}

Correlation coefficients were computed for every possible pair of the ten channels for each period. We can compute the $95 \%$ confidence level interval $R \pm\left[2\left(1-R^{2}\right) /(n)^{\frac{1}{2}}\right]$ for a correlation coefficient $R$ and a number $n$ of data if the distributions are assumed to be Gaussian (Hald, 1967). This is rather the case for the brightness temperatures. For example, with $n=3000$ and $R=0.9$, the interval is $0.9 \pm 0.007$. Results are very similar for the four periods and only November data are given in Table 2. There is a great deal of redundancy between variables, the most independent being the $6.6 \mathrm{GHz}$.

One advantage of this redundancy is that we can neglect the $21 \mathrm{GHz}$ channel without losing any important information. From multiple-regression analysis, we computed that the eight other channels explained $99 \%$ of the variance of the $21 \mathrm{GHz} \mathrm{H}$ (horizontal polarization) and $95 \%$ of variance of the $21 \mathrm{GHz} \mathrm{V}$ (vertical polarization). Due to the known drift on this channel, we decided to neglect it for the rest of the study. However, all the computations were repeated with this channel as a check and no major changes were observed. For simplicity, we will continue to refer to the $37 \mathrm{GHz}$ channels as channels 9 and 10 .

For multiple linear regression, a stepwise regression routine was used to select independent variables in the order of their importance and to enter them in a multipleregression model. The criterion of importance is based on the reduction of the sum of the squares, and the independent variable providing the largest reduction in a given step is entered in the regression.

This analysis was used to determine how much of the total variance is explained by $N$ channels $(N<8$ without the $21 \mathrm{GHz}$ ). If $V_{j}$ is the variance of data for channel $j$, the total variance $V_{\text {total }}$ is defined as:

$$
V_{\text {total }}=\sum_{j=1}^{10} V_{j} \quad j \neq 7,8 .
$$

With $N$ channels there is a number $N C$ of possible combinations. Using linear regression, combination $i$ explains $P_{i j} \%$ of the variance of channel $j$. The percentage of total variance explained by $N$ channels is then:

$$
P N=\frac{\sum_{i=1}^{\mathrm{NC}} \sum_{j=1}^{10} P_{i j} V_{j}}{V_{\text {total }}} \quad j \neq 7,8 .
$$

As for the correlation matrix, very similar results are found for the four periods. Results for November data only are presented.

When using one channel $(N=1$, Table 3$)$, the variability is very small from one channel to another. Channel $2(6.6 \mathrm{GHz} \mathrm{V})$ is the worst estimator of the other channels but nevertheless explains at least $92.5 \%$ of the variance.

With two channels $(N=2$, Table 3$)$, there is more variability. The worst estimator doublets $(<95 \%$ explained variance) are the vertical polarization doublets. The best estimators ( $>97 \%$ ) are the low-frequency, horizontal polarization doublets. We note also that channel $3(10 \mathrm{GHz} \mathrm{H})$ used with any other channel is a good estimator.

Not all the results are given for $N=3$ channels because of the large number of combinations. The percentage of explained variance varies from 97.3 to $99.4 \%$. The worst triplets are the high-frequency ones: triplets $(5-9-10)$ and $(6-9-10)$. Thirty-three triplets out of the total possible 56 combinations explain more than $99 \%$ of the total variance.

The percentage of variance explained by four channels ranges from 98.2 (quadruplet (4-6-9-10)) to $99.7 \%$ with 51 quadruplets out of the total number 70 above $99.5 \%$

In conclusion, the low-frequency channels $(\leq 10 \mathrm{GHz})$ are better estimators of the other channels than the highfrequency ones and any useful combination should include at least one of them. There is in any case a great deal of redundancy between variables and one should not expect to obtain as many different geophysical parameters as SMMR channels. And, finally, there is a remarkable stability of results throughout the year. 
Table 3. Percentage of total variance explained by $\mathcal{N}=1$ or $\mathcal{N}=2$ channels, November data

\begin{tabular}{|c|c|c|c|c|c|c|c|c|}
\hline 1 & 2 & 3 & 4 & 5 & 6 & 9 & 10 & Channel \\
\hline 94.0 & 92.5 & 94.9 & 93.3 & 94.1 & 93.3 & 93.0 & 93.0 & \\
\hline \multicolumn{9}{|c|}{$\mathcal{N}=2$} \\
\hline 1 & 2 & 3 & 4 & 5 & 6 & 9 & 10 & Channel \\
\hline \multirow[t]{8}{*}{-} & 96.4 & 97.5 & 97.0 & 98.0 & 96.7 & 96.6 & 96.5 & 1 \\
\hline & - & 97.2 & 94.8 & 96.5 & 94.8 & 94.7 & 94.5 & 2 \\
\hline & & - & 97.6 & 97.7 & 97.3 & 97.1 & 97.2 & 3 \\
\hline & & & - & 96.8 & 94.6 & 95.3 & 94.8 & 4 \\
\hline & & & & - & 96.4 & 96.3 & 96.6 & 5 \\
\hline & & & & & - & 95.0 & 94.7 & 6 \\
\hline & & & & & & - & 94.7 & 9 \\
\hline & & & & & & & - & 10 \\
\hline
\end{tabular}

\section{Principal-component analysis}

The aim of the principal-component analysis is to determine which linear combinations of channels explain the most variance of a data set. The combinations are chosen to be orthogonal or independent in the $N$-dimension space, $N$ being the number of components.

This analysis was conducted without the $21 \mathrm{GHz}$ channel as discussed previously. Therefore, we obtain eight principal components $Y_{i}$.

$$
Y_{i}=\sum_{j=1}^{10} a_{i j} T B_{j} \quad i=1,8 ; j \neq 7,8
$$

where $T B_{j}$ is the brightness temperature in channel $j$ and the $a_{i j}$ values are the weights of the principal components. If we consider the cloud of data points in the eight-dimensional space $T B$, the $a_{1 j}$ are chosen so that $Y 1$ lies in the direction of the maximum variance, the $a_{2 j}$ so that $Y 2$ lies in the direction of the maximum variance orthogonal to $Y 1$, and so on.

The weights $a_{i j}$ are given in Figure 2. The components are very stable in time and, as there is no difference between different periods, the percentage of total variance explained by each component is given on the same figure for November data only.

The first component $Y 1$ explains most of the variance and therefore contains the most information concerning the ice sheet. The reason is that the two main parameters (temperature and accumulation) affecting the brightness temperature are positively correlated and have the same effect on the measure. Low temperature means low accumulation, which in turn induces low emissivity and therefore low brightness temperature.

The total variance for November data is $2245 K^{2}$, so
$0.1 \%$ represents only $2 K^{2}$, which is close to the $1 K^{2}$ of SMMR instrument noise estimated by Gloersen and Barath (1977). Therefore, we conclude that the variances explained by the components $Y 4$ through $Y 8$ (Fig. 2) are too close to the instrument noise to derive any geophysical meaning. Another reason is that, if we use the $21 \mathrm{GHz}$ channels, differences appear only with the fourth component. Hence, in the following, we will consider only the first three components.

As the components $Y 2$ and $Y 3$ are very stable with time, and as the amount of variance they explain is greater than the instrument noise, they should have some geophysical meaning related to the ice-sheet surface. $Y 2$ reflects mainly the polarization difference at the lower frequencies. $Y 3$ is mostly related to the frequency gradient between 18 and $6.6 \mathrm{GHz}$. The importance of the lower-frequency channels over the ice sheet is stressed. Channel $9(37 \mathrm{GHz} \mathrm{H})$ which has a low weight for $Y 3$ and channel $10(37 \mathrm{GHz}$ V) which has a low weight for $Y 3$ have a slightly different behaviour than other frequencies. In the remainder of the text we will refer to $Y 2$ as the polarization difference and to $Y 3$ as the frequency gradient.

The $Y 2$ and $Y 3$ scores have been computed for November for each $100 \mathrm{~km} \times 100 \mathrm{~km}$ cell of the grid defined in the preceding section. Figure 3 gives the isocontours of the computed scores. The same plots have been drawn for the four periods and their patterns are very similar. The absolute values differ for $Y 3$ because the frequency gradient is dependent on the temperature profile which is different in summer than in winter. For the polarization difference $Y 2$, even the absolute values are constant throughout the year. This has also been noted by Zhang and others (1989) from spectral signa- 


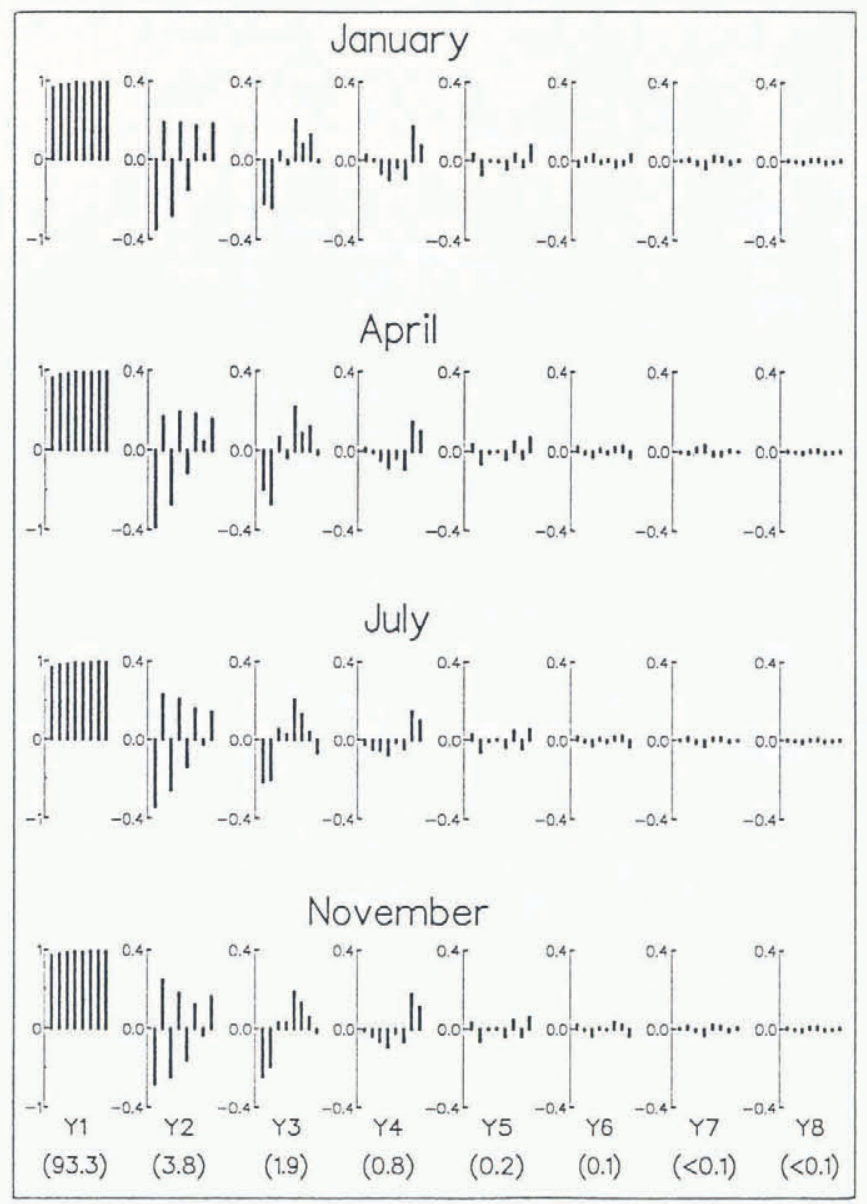

Fig. 2. Weights of principal components Y1 through Y8. For each component the bar farthest left corresponds to channel 1, the bar farthest right corresponds to channel 10 ; channels 7 and 8 are not used. There are two difference scales: one for $Y 1$ and another for Y2 through Y8. The percentage of the total variance explained by each principal component is given in parentheses for November data.

tures obtained over different sites of the central part of the Greenland ice sheet. We have independently checked the minor effect of the temperature on the polarization difference by using a snow-emissivity model based on the strong-fluctuation theory (Stogryn, 1986).

From the $Y 2$ and $Y 3$ spatial distribution, some areas can be selected which exhibit a marked microwave signature. Four areas have been selected as examples which we refer to in the discussion. They are noted A, B, C and $\mathrm{D}$ on the maps and their signatures are given on Figure 4 for the month of November. The signatures for other periods are very similar with only slight differences for the frequency gradient. The polarization differences are almost the same.

\section{COMPARISON WITH GEOPHYSICAL DATA}

The sets of data we use here are the gridded geophysical and SMMR data. The size of each cell is $100 \mathrm{~km} \times 100 \mathrm{~km}$ and the number of cells involved is about 1000 depending on the period.

\section{Correlation}

Simple cross-correlation coefficients have been computed between geophysical and SMMR data (Table 4). As stated above, if we assume the distributions of variables to be Gaussian, the $95 \%$ confidence level interval is $R+\left[2\left(1-R^{2}\right) /(n)^{\frac{1}{2}}\right]$ for a correlation coefficient $R$ and a number $n$ of data. Note that the accumulation-rate distribution is very dissymmetric over Antarctica and that we therefore have to be cautious when using confidence intervals for this variable. The distributions of the surface temperatures, the brightness temperatures and even the natural logarithm of the accumulation rates are almost Gaussian.

As we said previously, there are some uncertainties on snow accumulation-rate data. Therefore, to check
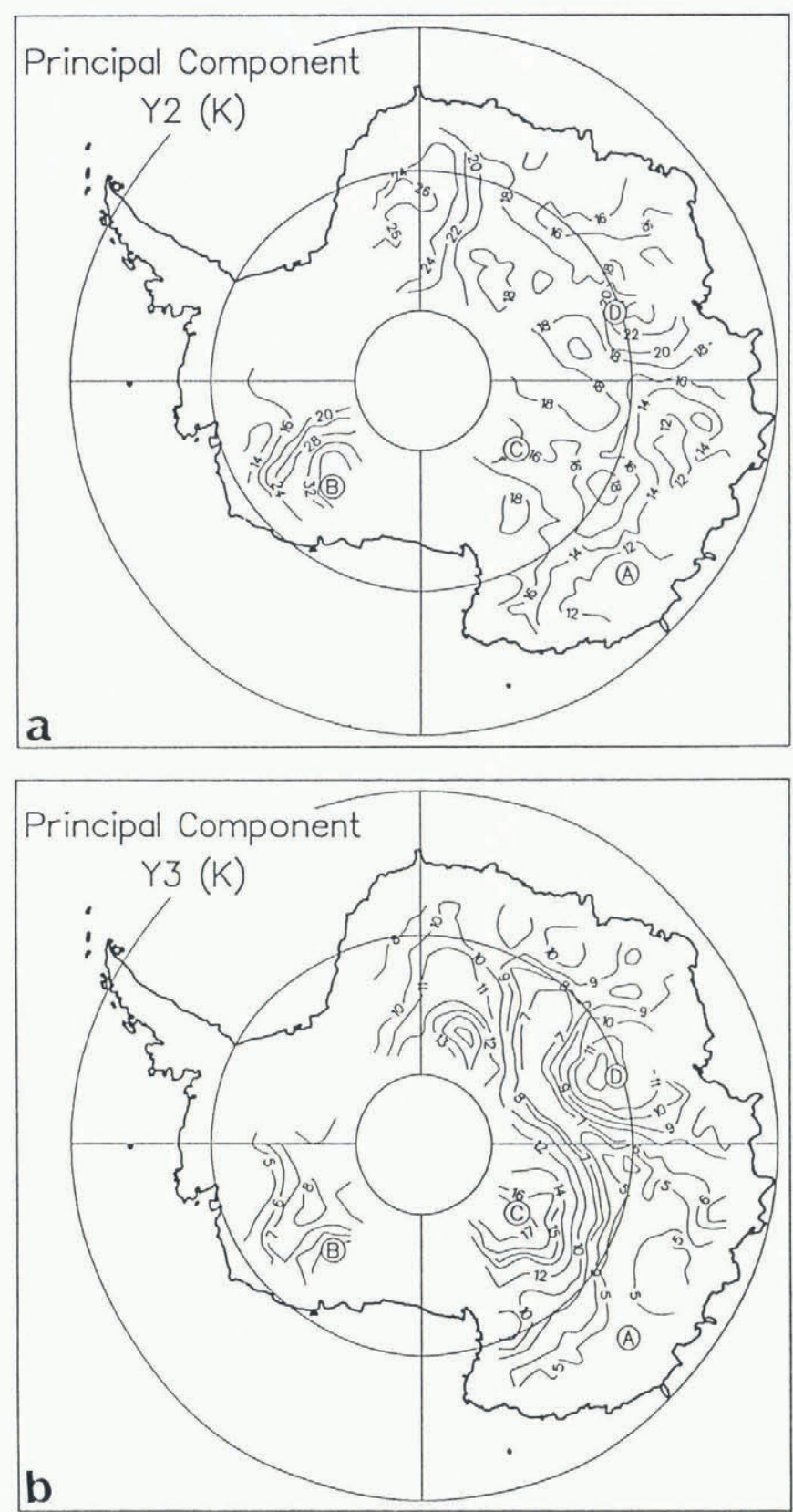

Fig. 3. Y2 and Y3 principal-component scores over Antarctica for November 1981 data. As Y3 scores are always negative, only absolute values are given. Y2 reflects the polarization difference and Y3 the frequency gradient. The labelled areas are the same as in Figure 1. 

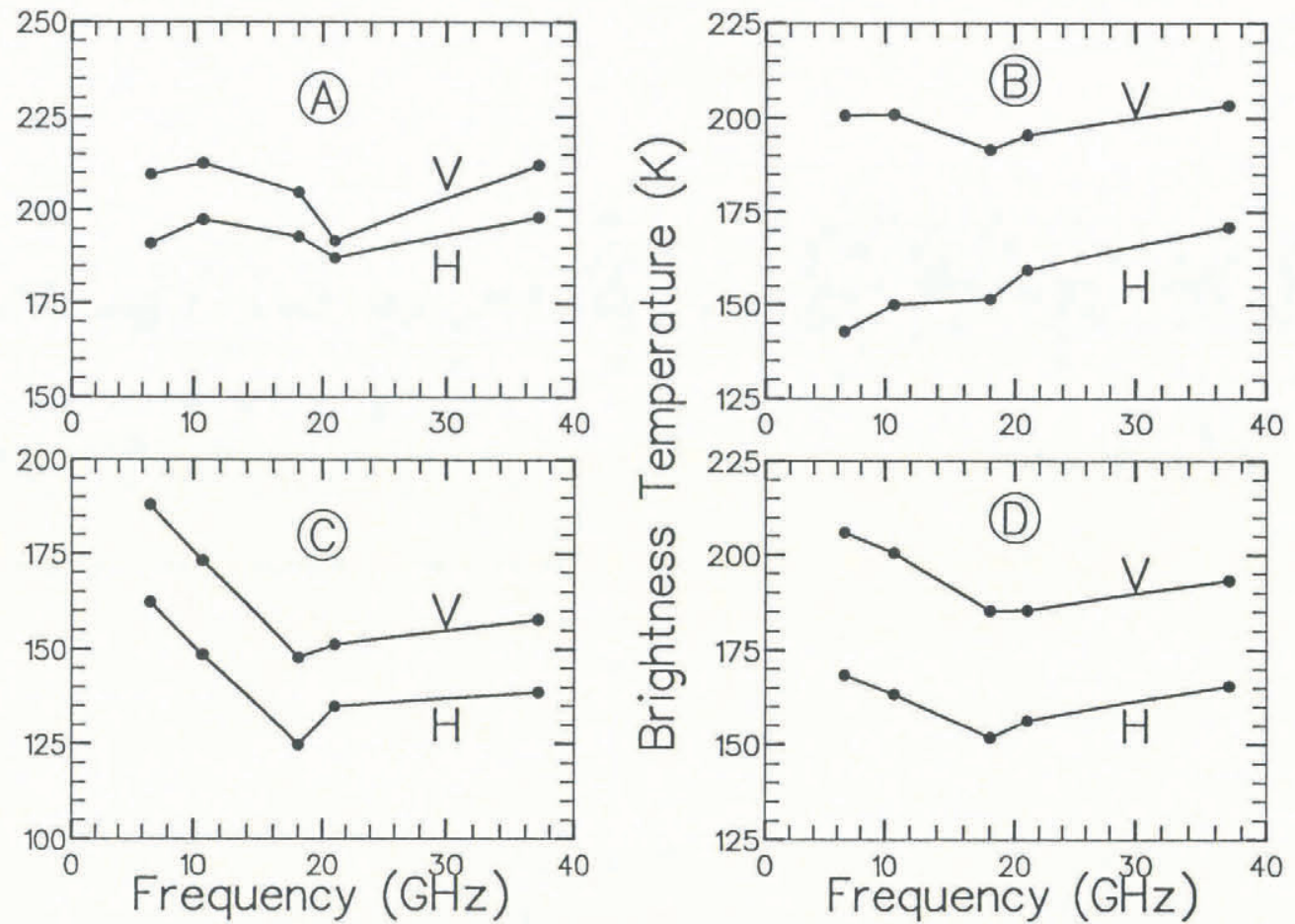

Fig. 4. Some selected microwave signatures on the Antarctic ice sheet. Letters refer to areas defined in Figures 1 and 3. Note the curious behaviour of the $21 \mathrm{GHz}$ channels for area $A$, possibly due to a malfunction of these channels.

the correlation results, we have also used a set of data to which some white noise was added. The white noise is computed as a random number whose mean is zero and variance is $50 \%$ of the variance of the original data set. That means that we consider a potential relative error of $50 \%$ on accumulation rates. The main conclusions we derive from correlations with accumulation-rate data will be checked using this white-noise-added data set.

Very good correlations are obtained with all channels every time. There is a strong correlation between the $6.6 \mathrm{GHz} \mathrm{V}$ channel and the mean annual temperature and, in general, vertically polarized channels are better correlated with temperature than horizontally polarized channels. The accumulation rate is better correlated with the $37 \mathrm{GHz}$ channels, and this is true whether using the white-noise-added data set or not. The better correlation between $T B$ and $\ln (A)$ than between $T B$ and
$A$ is coherent with the exponential shape of the relation given by Rotman and others (1982) to derive snowaccumulation rate from brightness temperature.

The correlation found between accumulation and temperature $(R=0.74)$ is similar to the one given by Musynski and Birchfield (1985) in their parameterization of accumulation rates over Antarctica. The better correlation with the logarithm of the accumulation rate $(R=0.83)$ is due to the fact that the accumulation is roughly proportional to the water-vapour saturation pressure in the inversion layer (Robin, 1977), which is itself an exponential function of the temperature following the Clausius-Clapeyron law.

Although the polarization difference $Y 2$ is poorly correlated both with the temperature and the accumulation, the signs of the correlation are opposite. This is worth noting because the polarization difference therefore pro-

Table 4. Correlation coefficients between brightness temperatures $T B_{j}$ in channel $j$ or principal components $Y_{i}$, and mean surface temperature $T$ or accumulation rate over the Antarctic ice sheet. $r 1$ is an almost equal combination of all the channels, $Y 2$ reflects the polarization and $Y 3$ the frequency gradient

\begin{tabular}{lcccccccccccc}
\hline & 6.6 & 6.6 & 10 & 10 & 18 & 18 & 37 & 37 & $\Upsilon 1$ & $\Upsilon 2$ & $\Upsilon 3$ & $T$ \\
& $\mathrm{H}$ & $\mathrm{V}$ & $\mathrm{H}$ & $\mathrm{V}$ & $\mathrm{H}$ & $\mathrm{V}$ & $\mathrm{H}$ & $\mathrm{V}$ & & & & \\
\hline$T$ & 0.65 & 0.88 & 0.62 & 0.80 & 0.63 & 0.75 & 0.75 & 0.83 & 0.76 & 0.21 & 0.33 & - \\
$A$ & 0.77 & 0.79 & 0.79 & 0.80 & 0.79 & 0.79 & 0.82 & 0.80 & 0.82 & -0.24 & 0.53 & 0.74 \\
$\ln (A)$ & 0.81 & 0.89 & 0.84 & 0.88 & 0.85 & 0.87 & 0.89 & 0.91 & 0.89 & -0.15 & 0.58 & 0.83 \\
\hline
\end{tabular}


Table 5. Percentage (\%) of variance explained and RMS at each step of linear regressions between temperature or accumulation and the SMMR brightness temperatures for four periods in 1981

(1) Dependent variable is $\ln$ (accumulation)

\begin{tabular}{lcclcc} 
Fanuary & \multicolumn{5}{c}{ April } \\
Channel & 9 & 2 & Channel & 9 & 2 \\
$\%$ & 82 & 1 & $\%$ & 81 & 2 \\
RMS & 0.38 & 0.36 & RMS & 0.38 & 0.36 \\
July & & & & & \\
Channel & 10 & 2 & November & \\
$\%$ & 79 & 1 & Channel & 10 & 9 \\
RMS & 0.41 & 0.40 & RMS & 82 & 0.38 \\
\end{tabular}

(2) Dependent variable is temperature ( $R M S$ in $K$ )

\begin{tabular}{|c|c|c|c|c|c|c|c|c|c|}
\hline Januar & & & & & April & & & & \\
\hline Chann & 12 & 3 & 9 & 10 & Chann & 12 & 3 & 9 & 6 \\
\hline$\%$ & 79 & 5 & 2 & 2 & $\%$ & 78 & 5 & 2 & 3 \\
\hline RMS & 5.1 & 4.5 & 4.2 & 3.9 & RMS & 5.1 & 4.4 & 4.2 & 3.7 \\
\hline July & & & & & Novem & & & & \\
\hline Chann & & 3 & 9 & 6 & Chann & 12 & 3 & 9 & 6 \\
\hline$\%$ & 79 & 7 & 2 & 2 & $\%$ & 78 & 7 & 2 & 2 \\
\hline RMS & 5.1 & 4.4 & 4.2 & 3.7 & RMS & 5.2 & 4.2 & 3.9 & 3.6 \\
\hline
\end{tabular}

vides an opportunity to discriminate the effect of temperature and accumulation on the brightness temperatures which is usually difficult to do because they are highly correlated. Using the white-noise-added set of data does not modify this result, which will be discussed later on.

\section{Linear regression analysis}

As a complement to the correlation analysis, we have computed linear regressions between SMMR and geophysical data using the stepwise routine. For the temperature $(T)$ and the accumulation rate $(A)$, the best regressions have been computed using either all the channels from one period (Table 5) or all the channels from all periods (Table 6). As previously, the $21 \mathrm{GHz}$ channels are omitted. Only the channels explaining more than $1 \%$ of the variance of the geophysical data are given.
The RMS (root mean square) has been computed as:

$$
\mathrm{RMS}^{2}=\sum_{i=1}^{n}\left(X \text { est }_{i}-X_{i}\right)^{2} /(n-1),
$$

$X_{i}$ being the observed measure (either $\ln (A)$ or $T$ ), $X$ est $_{i}$ the corresponding estimated value, and $n$ the total number of observations.

Concerning the accumulation rate, we note that using many channels does not improve the regression significantly even with the four periods together. Channels 9 or 10 were used preferably depending on the period.

For the temperature, some improvement is obtained when using four channels instead of one. Results are very similar throughout the year. The use of all the four 
Table 6. Percentage (\%) of variance explained and RMS at each step of linear regressions between temperature or accumulation and all the SMMR brightness temperatures from four periods in the year

(1) Dependent variable is $\ln$ (accumulation)

$\begin{array}{lrr}\text { Day } & 22 & 305 \\ \text { Channel } & 9 & 2 \\ \% & 82 & 2\end{array}$

(2) Dependent variable is temperature ( $R M S$ in $K$ )

Day

22

Channel

2

80

RMS
305

3

6

3.9
305

9

2

3.5
305

5

1

3.4 data periods does not improve the results significantly and the three most important channels are surprisingly the same when one or four periods are used. The multichannel approach is more conclusive than the multidate approach.

Although our aim is not to parameterize the temperature or the accumulation rate, our results can be compared with those obtained by Fortuin and Oerlemans (1990). Using selected ground measurements, they found that $81 \%$ of the temperature variance is explained by elevation and latitude while only $40 \%$ of the surface mass-balance variance can be explained by the saturation vapour pressure of the free atmosphere, the surface slope and the surface shape (convergence or divergence). Therefore, the use of brightness temperature does not significantly improve the parameterization of the surface temperature but gives better results for the accumulation rate.

\section{DISCUSSION}

Although $Y 1$ explains most of the variance, this principal component does not seem to add more information than when using single channels. It is an almost equal combination of all the channels which are all very closely related to accumulation rate and temperature but it does not help in discriminating between these two closely correlated quantities. Apart from $Y 1, Y 2$ and $Y 3$ explain a non-negligible part of the variance and seem to have a geophysical significance that we will now discuss.

$Y 2$ and $Y 3$ reflect the polarization and the freqeuncy gradient which are dependent on the structure of the firn. This structure depends either on the precipitation mechanism (quantitatively and qualitatively) and/or the firn evolution (stratification, depth-hoar formation, etc.).

It is therefore important to examine the main processes governing the accumulation of snow in Antarctica (Bromwich, 1988). The amount of precipitation is directly linked to the amount of atmospheric water vapour and therefore depends on the water-vapour fluxes towards the Antarctic ice sheet. The water vapour is therefore condensed according to three major processes:

(1) Air cooling by orographic lifting over the marginal ice slopes.

(2) Radiative cooling over the continental area.
(3) Downward transport of air and moisture, or subsidence. This latter process is relatively more important on the central plateau.

The zonal differences in annual moisture transport along the coast of East Antarctica have been given by Bromwich (1988). It is surprising to note that areas of water-vapour output correspond to high $Y 2$ scores or large polarization difference (between $0^{\circ}$ and $10^{\circ} \mathrm{E}$ and $60^{\circ}$ to $70^{\circ} \mathrm{E}$ ), and input to low $Y 2$ scores or small polarization difference (between $10^{\circ}$ and $60^{\circ} \mathrm{E}, 70^{\circ}$ and $120^{\circ} \mathrm{E}$ ). A similar study has not been done for West Antarctica but there is also some evidence of low water content with respect to the temperature over the Byrd Station area (area B on the maps). Some cyclone trajectories given by Rubin and Giovinetto (1962) indicate that air arriving over the Byrd Station area comes from the north, therefore passing over the Executive Commit-

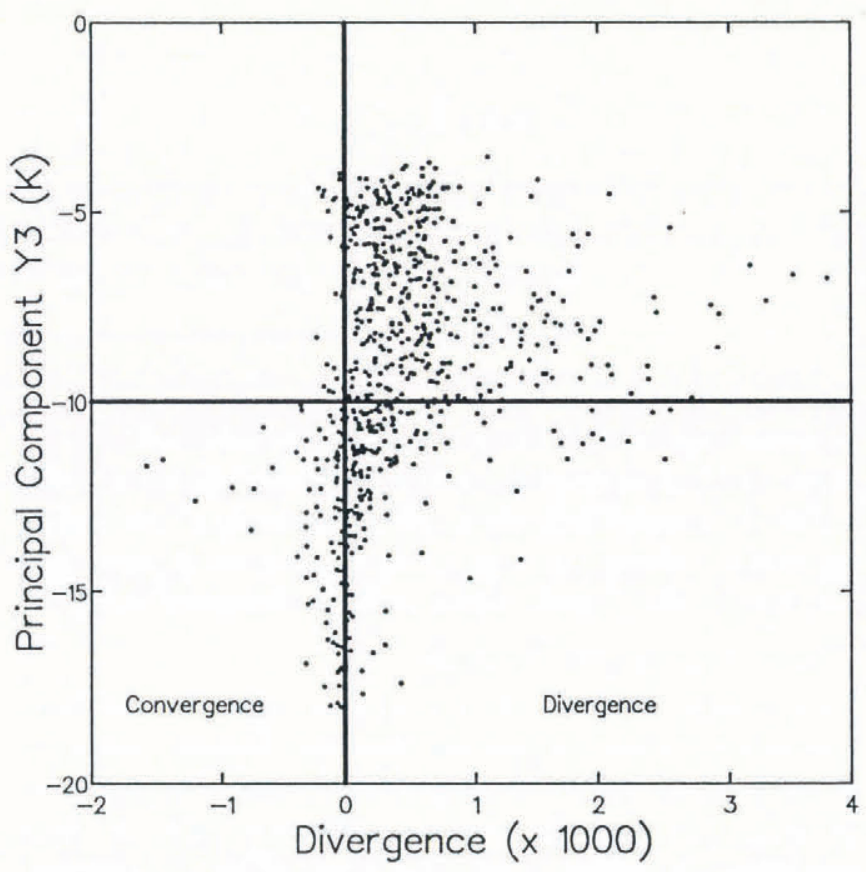

Fig. 5. Scatter plot of Y3 scores (frequency gradient) against the divergence. Each dot represents one grid point, the grid spacing being $100 \mathrm{~km}$. 
tee Range (elevations up to $3000 \mathrm{~m}$ ). High precipitation occurs on these mountains, leaving dried air in the subsequent southward-moving air masses. Schwerdtfeger (1970) also noted that $70 \%$ of the $700 \mathrm{mbar}$ level winds are from the north. This level is the most important when looking at precipitation because the almost permanent inversion regime over Antarctica keeps it warmer and therefore moister than the lower levels of the atmosphere.

The polarization difference therefore appears to be related to a deficit of the atmospheric water-vapour content, i.e. of the precipitation, with respect to the temperature. This can be assessed by the following simple computation.

A linear regression is computed between the logarithm of the accumulation rate and the temperature, giving an estimated accumulation rate:

$$
\ln (\text { Aest })=0.06695 T-13.413
$$

with Aest: estimated accumulation rate $\left(\right.$ gyear $\left.^{-1} \mathrm{~cm}^{-2}\right)$; $\mathrm{T}$ : annual mean temperature $(\mathrm{K})$.

Let Dif be the difference between Aest and the accumulation rate $A$ as given in the original data set:

$$
\text { Dif }=A \text { est }-A \text {. }
$$

Dif is computed for each cell of the grid and then correlated with $Y 2$. The cross-correlation coefficient is $R=-0.57$. Using the white-noise-added data set, we still found $R=-0.38$. That means that the polarization difference $Y 2$ is an indicator of the departure of $A$ est from the linear parameterization. In other words, the polarization difference is large when $A$ is low with respect to $T$. The main areas concerned are the Ross sector of West Antarctica (area B, Fig. 3), the Lambert Glacier drainage basin (area D), and the Dronning Maud Land area. We suppose that a relatively high temperature induces different snow-grain ageing and stratification which modifies the polarization. Rott (1989) reached the same conclusion.

On a smaller scale, Remy and others (1991) found a strong correlation between polarization and katabatic flow areas in East Antarctica. The effect of wind there is supposed to modify the surface roughness, a higher roughness inducing a smaller polarization difference. This cannot be confirmed or denied by our largescale study. We note, however, that vertically polarized brightness temperatures are better correlated with surface temperature or accumulation than the horizontally polarized ones. As the Fresnel reflection coefficient is close to zero for vertical polarization at the SMMR incidence angle $\left(50^{\circ}\right)$, an effect other than temperature or accumulation, i.e. surface roughness, could influence the horizontally polarized measures only, but it could also be the effect of stratigraphy.

Concerning the frequency gradient $Y 3$, some resemblance can be found between its spatial pattern (Fig. 3) and the localization of convergence and divergence areas on the elevation map (Fig. 1). The absolute value of the frequency gradient is low on domes and ridges, i.e. diverging areas, and is relatively high in converging areas such as the Lambert Glacier drainage basin (area D,
Fig. 3). This is true only for the central plateau area above $2000 \mathrm{~m}$. Divergence can be computed using the method of Jenssen and others (1985):

$$
\text { Div }=(u \text { East }-u \text { West }+u \text { North }-u \text { South }) / 2 D
$$

where the $u$ s are the differences of elevation in the four directions between a grid point and its neighbours, and $D$ the grid spacing $(100 \mathrm{~km})$. The $Y 3$ scores (frequency gradient) are plotted against the divergencies Div in Figure 3. Low $Y 3$ absolute values are related to diverging areas when almost all the converging areas are associated with large negative $Y 3$ scores under a threshold we have set at $Y 3=-10 \mathrm{~K}$. We also note that the frequency gradient $Y 3$ is highly correlated with the variable Dif as defined above $(R=0.47)$, indicating that small frequency gradients are related to areas where the accumulation is high with respect to the temperature. This must be related to the positive correlation between the divergence and the accumulation rate which is due to the subsidence process as noted by Fortuin and others (1990). Over diverging areas, there are more downward air fluxes. As the lower atmosphere is cooler than the inversion level, subsidence induces condensation and therefore precipitation. As the frequency gradient is dependent on the grain-size and shape (Reber and others, 1987), $Y 3$ could reflect a different precipitation process (subsidence), producing a different type of snow crystal.

\section{CONCLUSION}

There is considerable redundancy between the ten channels of the SMMR instrument for measurements over the Antarctic ice sheet and more than $99.5 \%$ of the total variance is explained by many different combinations of only four channels. The low-frequency channels $(\leq 10 \mathrm{GHz})$ are nevertheless of a somewhat greater importance than the high-frequency channels.

Although all the channels are very well correlated with the mean surface temperature $(T)$ and the logarithm of the accumulation rate $(\ln (A))$, the $6.6 \mathrm{GHz} \mathrm{V}$ is better correlated with $T$ and the $37 \mathrm{GHz}$ (H or V) with $\ln (A)$. The results are very similar for different periods of the year and the use of multi-temporal data does not improve the linear parameterization of $T$ or $A$.

The dependence of $\ln (A)$ on $T$ and their identical effect on the brightness temperatures is the main reason that the first principal component $Y 1$, which is an almost equal combination of all channels, explains more than $93 \%$ of the total variance. Two other principal components, $Y 2$ and $Y 3$, explain a non-negligible part of the variance, and their spatial distributions show an interesting pattern over the ice sheet.

The second principal component, $Y 2$, which reflects the polarization, indicates areas where the accumulation rate is low with respect to the temperature, due to a lower atmospheric water-vapour content. $Y 3$ is linked to the frequency gradient, and could reflect the importance of the subsidence effect over diverging areas such as domes or ridges. This process is more intensive over the central plateau area.

Having used global data sets, the accumulation-rate data are uncertain, and more accurate ground data are 
needed for a parameterization. In any case, it is clear that the microwave signature of the ice-sheet surface will improve our understanding of precipitation processes, i.e. interactions between climate and ice sheet, and also provide information on the snow structure depending on where it has been deposited, useful for ice-core interpretation.

More studies are needed to relate the microwave signature to the firn structure. This large-scale study could serve as a guide for future research to select areas for ground-truth data collection or to be sampled by other sensors such as the active microwave instrument to be installed on the European satellite ERS1.

\section{ACKNOWLEDGEMENTS}

This work was funded by ATP Teledetection (contract 87/CNES/1254). We are grateful to Dr D. Han, Dr P. Gloersen, Dr P.H. Hwang from NASA/GSFC for providing the SMMR data, and to Dr D. Jenssen from the Meteorology Department, University of Melbourne, for the geophysical data sets. We thank C. Ritz from LGGE for valuable discussions.

\section{REFERENCES}

Bromwich, D. H. 1988. Snowfall in high southern latitudes. Rev. Geophys., 26(1), 149-168.

Budd, W. F. and I. N. Smith. 1982. Large-scale numerical modelling of the Antarctic ice sheet. Ann. Glaciol., 3, 42-49.

Chang, T. C., P. Gloersen, T. Schmugge, T. T. Wilheit and H. J. Zwally. 1976. Microwave emission from snow and glacier ice. J. Glaciol., 16(74), 23-39.

Chang, A. T. C., B. J. Choudhury and P. Gloersen. 1980. Microwave brightness of polar firn as measured by Nimbus 5 and 6 ESMR. J. Glaciol., 25(91), 85-91.

Drewry, D. J., ed. 1983. The surface of the Antarctic ice sheet. In Drewry, D. J., ed. Antarctica: glaciological and geophysical folio. Cambridge, University of Cambridge. Scott Polar Research Institute, Sheet 2.

Fortuin, J. P.F. and J. Oerlemans. 1990. Parameterization of the annual surface temperature and mass balance of Antarctica. Ann. Glaciol., 14, 78-84.

$\mathrm{Fu}$, C. C., D. Han, S. T. Kim and P. Gloersen. 1988. User's guide for the Nimbus 7 Multichannel Microwave Radiometer (SMMR), Cell-All Tape. Greenbelt, MD, Goddard Space Flight Center. (NASA Reference Publication 1210.)

Giovinetto, M.B. and C. Bull. 1987. Summary and analyses of surface mass balance compilations for Antarctica, 1960-1985. Columbus, OH, Ohio State University. Byrd Polar Research Center. (Report 1.)

Gloersen, P. and F. T. Barath. 1977. A Scanning Multichannel Microwave Radiometer for Nimbus-G and Seasat-A. IEEE J. Oceanic Eng., OE2 (2), 172-178.

Gloersen, P. and W.J. Campbell. 1988. Variations in the Arctic, Antarctic, and global sea ice covers during 1978-1987 as observed with the Nimbus 7 Scanning Multichannel Microwave Radiometer. J. Geophys. Res., 93(C9), 10,666-10,674.

Gloersen, P., T. T. Wilheit, T. C. Chang, W. Nordberg and W. J. Campbell. 1974. Microwave maps of the polar ice of the Earth. Bull. Am. Meteorol. Soc., 55(12), 1442-1448.

Gow, A.J. 1969. On the rates of growth of grains and crystals in south polar firn. J. Glaciol., 8(53), 241252.

Hald, A. 1967. Statistical theory with engineering applications. New York, John Wiley and Sons.

Jenssen, D., W. F. Budd, I. N. Smith and U. Radok. 1985. On the surging potential of polar ice streams. Part II. Ice streams and physical characteristics of the Ross Sea drainage basin, West Antarctica. Boulder, CO, University of Colorado. Cooperative Institute for Research in Environmental Sciences/Parkville, Victoria, University of Melbourne. Meteorology Department.

Kunzi, K.F., S. Patil and H. Rott. 1982. Snow-cover parameters retrieved from Nimbus-7 Scanning Multichannel Microwave Radiometer (SMMR) data. IEEE Trans. Geosci. Remote Sensing, GE-20(4), 452467.

Madrid, C. R., ed. 1978. The Nimbus-7 user's guide. Greenbelt, MD, Goddard Space Flight Center.

Mätzler, C. 1987. Applications of the interaction of the microwaves with the natural snow cover. Remote Sensing Rev., 2(2), 259-387.

Muszynski, I. and G. E. Birchfield. 1985. The dependence of Antarctic accumulation rates on surface temperature and elevation. Tellus, 37A, 204-208.

Radok, U., T. J. Brown, D. Jenssen, I. N. Smith and W.F. Budd. 1986. On the surging potential of polar ice streams. Part IV. Antarctic ice accumulation basins and their main discharge regions. Boulder, CO, University of Colorado. Cooperative Institute for Research in Environmental Sciences; Parkville, Victoria. University of Melbourne. Meteorology Department.

Reber, B., C. Mätzler and E. Schanda. 1987. Microwave signatures of snow crusts. Modelling and measurements. Int. J. Remote Sensing, 8(11), 1649-1665.

Remy, F. and J. F. Minster. 1991. A comparison between active and passive microwave measurements of the Antarctic ice sheet and their association with the surface katabatic winds. J. Glaciol., 37 (125), 3-10.

Robin, G. de Q. 1977. Ice cores and climatic change. Philos. Trans. R. Soc. London., Ser. B., 280(972), 143-168.

Rothrock, D. A., D. R. Thomas and A.S. Thorndike. 1988. Principal component analysis of satellite passive microwave data over sea ice. J. Geophys. Res., 93(C3), 2321-2332.

Rotman, S.R., A.D. Fisher and D.H. Staelin. 1981. Analysis of multiple-angle microwave observations of snow and ice using cluster-analysis techniques. J. Glaciol., 27(95), 89-97.

Rotman, S. R., A. D. Fisher and D. H. Staelin. 1982. Inversion for physical characteristics of snow using passive radiometric observations. J. Glaciol., 28(98), 179-185.

Rott, H. 1989. Multispectral microwave signatures of the Antarctic ice sheet; microwave radiometry and remote sensing applications. In Pampaloni, P., ed. Proceed- 
ings of the specialist meeting held in Florence, Italy, 9-11 March 1988.

Rubin, M. J. and M. B. Giovinetto. 1962. Snow accumulation in central West Antarctica as related to atmospheric and topographic factors. J. Geophys. Res., 67(13), 5163-5170.

Schwerdtfeger, W. 1970. The climate of the Antarctic. In Orvig, S., ed. Climates of the polar regions. Amsterdam, etc., Elsevier, 253-355. (World Survey of Climatology, Vol. 14.)

Zhang, H., L. T. Pedersen and P. Gudmandsen. 1989.
Microwave brightness temperatures of the Greenland ice sheet. Adv. Space Res., 9(1), 277-287.

Zwally, H.J. 1977. Microwave emissivity and accumulation rate of polar firn. J. Glaciol., 18(79), 195215.

Zwally, H. J. and P. Gloersen. 1977. Passive microwave images of the polar regions and research applications. Polar Rec., 18(116), 431-450.

The accuracy of references in the text and in this list is the responsibility of the authors, to whom queries should be addressed.

MS received 1 February 1990 and in revised form 23 July 1990 